\section{References}

1. Tamasyan G. Sh. The gradient methods for solving the Cauchy problem. Vestnik St. Petersburg University. Ser. 10, 2009, iss. 4, pp. 224-230 (in Russian).

2. Vasilyev L. V., Demyanov V. F. Nedifferenciruemaja optimizacija [Nondifferentiable optimization]. Moscow, Nauka, 1981. 384 p. (in Russian).

3. Kantorovich L. V., Akilov G. P. Funkcional'nyj analiz [Functional analysis]. Moscow, Nauka, 1977. 741 p. (in Russian).
4. Demyanov V. F. Usloviya ekstremuma i variacionnoe ischislenie [Extremum conditions and variation calculus]. Moscow, Vysshaya shkola, 2005. 335 p. (in Russian). 5. Vasilyev F. P. Metody optimizacii [Optimization methods]. Moscow, Faktorial Press, 2002. 824 p. (in Russian).

6. Arrowsmith D. K., Place C. M. Ordinary differential equations. A qualitative approach with applications. London, Chapman and Hall, 1982. 243 p.

\title{
УДК 512.5
}

\section{НОВЫЕ СВОЙСТВА ПОЧТИ НИЛЬПОТЕНТНОГО МНОГООБРАЗИЯ ЭКСПОНЕНТЫ ДВА}

\begin{abstract}
О. В. Шулежко
Аспирантка кафредры алгебро-геометрических вычислений, Ульяновский государственный университет, ol.shulezhko@gmail.com

В данной работе исследуются числовые характеристики почти нильпотентного многообразия экспоненты два, впервые построенного в статье [1]. Основным результатом данной работы является нахождение точных значений кратностей неприводимых модулей, входящих в разложение полилинейной части многообразия. В качестве следствия получены фрормулы для коразмерности и кодлины изучаемого многообразия.
\end{abstract}

Ключевые слова: многообразие, экспонента многообразия, коразмерность, кодлина.

Совокупность алгебр, в которых выполняется фиксированный набор тождеств, называется многообразием. Многообразие будем называть почти нильпотентным, если оно само не является нильпотентным, но каждое собственное его подмногообразие нильпотентно. Основой для работы послужила статья [1], в которой впервые был построен пример почти нильпотентного многообразия, рост которого экспоненциален. Более точно было доказано, что асимптотически последовательность коразмерностей этого многообразия ведет себя как $2^{n}$, т. е. так называемая, экспонента этого многообразия равна двум. Целью данной работы является вычисление основных числовых характеристик этого многообразия. Заметим, что так как в рассматриваемых алгебрах не предполагается выполнения тождества ассоциативности, то в произведениях следует следить за расстановкой скобок. Договоримся опускать скобки в случае их левонормированной расстановки, например, $x y z=(x y) z$.

Обозначим через $\Phi$ основное поле, которое на протяжении всей работы имеет нулевую характеристику. Все неопределяемые понятия можно найти в книге [2]. Для удобства читателей приведем определения основных понятий, которые используются в данной работе. В свободной алгебре многообразия $\mathbf{V}$ со счетным множеством свободных образующих $X=\left\{x_{0}, x_{1}, x_{2}, \ldots\right\}$ рассмотрим множество полилинейных элементов степени $n$ от $x_{1}, x_{2}, \ldots, x_{n}$. Они образуют векторное пространство $P_{n}(\mathbf{V})$, называемое полилинейной компонентой степени $n$ относительно свободной алгебры. Размерность этого пространства обозначим $c_{n}(\mathbf{V}), n=1,2, \ldots$ Хорошо известно, что полилинейную компоненту степени $n$ можно рассматривать как модуль над групповым кольцом $\Phi S_{n}$ симметрической группы $S_{n}$, задавая действие перестановки на индексах образующих. Известно, что с точностью до изоморфизма неприводимые $\Phi S_{n}$ модули можно описывать на языке разбиений и диаграмм Юнга. Разбиением числа $n$ называют набор целых положительных чисел $\lambda=\left(\lambda_{1}, \ldots, \lambda_{k}\right)$, при этом $\lambda_{1} \geq \cdots \geq \lambda_{k}>0$ и $n=\sum_{i=1}^{k} \lambda_{i}$. Разбиение $\lambda$ числа $n$ обозначают следующим образом: $\lambda \vdash n$. Для каждого такого разбиения $\lambda$ строится диаграмма Юнга, состоящая из $k$ строк, причем строка с номером $i$ должна содержать $\lambda_{i}$ клеток.

Так как характеристика основного поля равна нулю, то по теореме Машке полилинейную часть степени $n$ можно разложить в прямую сумму неприводимых подмодулей. Строение модуля $P_{n}(\mathbf{V})$ можно представить на «языке характеров». Рассмотрим разложение характера модуля $P_{n}(\mathbf{V})$ в целочисленную комбинацию неприводимых характеров:

$$
\chi_{n}(\mathbf{V})=\chi\left(\mathbf{P}_{\mathbf{n}}(\mathbf{V})\right)=\sum_{\lambda \vdash \mathbf{n}} \mathbf{m}_{\lambda}(\mathbf{V}) \chi_{\lambda},
$$


где $m_{\lambda}(\mathbf{V})$ - кратность неприводимого характера $\chi_{\lambda}$, отвечающего разбиению $\lambda$. Асимптотическое поведение размерности $c_{n}=c_{n}(\mathbf{V})$ пространства $P_{n}(\mathbf{V})$ определяет рост многообразия. Предел $\sqrt[n]{c_{n}(\mathbf{V})}$, в случае его существования, называется экспонентой многообразия и обозначается как еxp $\mathbf{V}$. Число слагаемых $l_{n}(\mathbf{V})=\sum_{\lambda \vdash \mathbf{n}} \mathbf{m}_{\lambda}$ в сумме (1) называют кодлиной многообразия.

Пусть теперь $Q_{n}(\mathbf{V})=\operatorname{span}\left\{\mathbf{x}_{\mathbf{0}} \mathbf{x}_{\sigma(\mathbf{1})} \ldots \mathbf{x}_{\sigma(\mathbf{n})} \mid \sigma \in \mathbf{S}_{\mathbf{n}}\right\}-$ пространство полилинейных левонормированных одночленов от $x_{0}, \ldots, x_{n}$, с $x_{0}$ в качестве самого левого сомножителя. Симметрическая группа $S_{n}$ действует на $Q_{n}(\mathbf{V})$ перестановкой индексов образующих $x_{1}, \ldots, x_{n}$, и пространство $Q_{n}(\mathbf{V})$ является $S_{n}$-модулем. Рассмотрим разложение его характера в сумму неприводимых

$$
\chi_{n}^{Q}(\mathbf{V})=\sum_{\lambda \vdash \mathbf{n}} \mathbf{m}_{\lambda}^{\mathbf{Q}}(\mathbf{V}) \chi_{\lambda}
$$

где $m_{\lambda}^{Q}(\mathbf{V})$ - кратность неприводимого характера $\chi_{\lambda}$ в характере $\chi_{n}^{Q}(\mathbf{V})$.

Договоримся использовать черту или волну над образующими для обозначения кососимметризации. Например,

$$
x_{0} \bar{x}_{1} \tilde{y}_{1} \tilde{y}_{2} \bar{x}_{2} \bar{x}_{3}=\sum_{p \in S_{3}, q \in S_{2}}(-1)^{p}(-1)^{q} x_{0} x_{p(1)} y_{q(1)} y_{q(2)} x_{p(2)} x_{p(3)},
$$

где $S_{m}$ - симметрическая группа, а $(-1)^{r}-$ четность перестановки $r$. Поясним процедуру альтернирования элемента по некоторым наборам образующих на следующем примере: результатом альтернирования монома $x_{0} x_{1} y_{1} x_{2} y_{2} y_{3} x_{3} x_{4}$ по наборам $x_{1}, x_{2}, x_{3}, x_{4}$ и $y_{1}, y_{2}, y_{3}$ является элемент $x_{0} \bar{x}_{1} \tilde{y}_{1} \bar{x}_{2} \tilde{y}_{2} \tilde{y}_{3} \bar{x}_{3} \bar{x}_{4}$.

Основным объектом в данной статье является алгебра $A$, построенная в работе [1]. Эта алгебра с одной бинарной билинейной операцией определяется тремя образующими элементами $a, b, z$ и следующими определяющими соотношениями:

1) $a^{2}=b^{2}=a b=b a=a z=b z=0$;

2) $\left(z w\left(R_{a}, R_{b}\right)\right)\left(z w^{\prime}\left(R_{a}, R_{b}\right)\right)=0$, для любых слов $w, w^{\prime}$ от $R_{a}$ и $R_{b}$;

3) $z\left(R_{a} R_{b}\right)^{k} R_{a} R_{b}+z\left(R_{a} R_{b}\right)^{k} R_{b} R_{a}=0, z\left(R_{a} R_{b}\right)^{k} R_{a}^{2}=z\left(R_{a} R_{b}\right)^{k} R_{b}^{2}=0$ для всех $k \geq 0$.

Поясним, что через $R_{c}$ обозначен оператор правого умножения на элемент $c$, причем символ отображения мы пишем справа от аргумента $d \in A$, то есть $d R_{c}=d c$. Удобство такого обозначения в том, что, например, $R_{c}^{3}$ - это степень линейного отображения, поэтому запись $d R_{c}^{3}$ является корректной и обозначает такое левонормированное произведение $d c c c$, которое нельзя записать как $d c^{3}$.

Для удобства читателей изложим некоторые результаты, полученные в статье [1]. Базис рассматриваемой алгебры $A$ состоит из элементов:

$$
a, b, z\left(R_{a} R_{b}\right)^{k}, z\left(R_{a} R_{b}\right)^{k} R_{a}, z\left(R_{a} R_{b}\right)^{k} R_{b}
$$

для $k \geq 0$. В алгебре $A$ выполняются следующие тождества:

$$
\begin{gathered}
x_{1}\left(x_{2} x_{3}\right) \equiv 0, \\
x_{0} x x x \equiv 0, \\
x_{0} x x y_{1} \ldots y_{2 s+1} y y \equiv 0 .
\end{gathered}
$$

Из тождества (3) следует, что только левонормированные многочлены относительно свободной алгебры могут иметь ненулевое значение в алгебре $A$.

Перейдем к изложению результатов, связанных с числовыми характеристиками многообразия $\operatorname{var} A$. В статье [1] получено следующее условие на кратности кохарактера:

$$
\begin{gathered}
\chi_{2 k+1}^{Q}(\operatorname{var} A)=2 \chi_{(k+1, k)}, \\
\chi_{2 k}^{Q}(\operatorname{var} A)=\alpha \chi_{(k, k)}+\chi_{(k+1, k-1)}, \quad \text { где } \quad \alpha=1 \quad \text { или } \quad \alpha=2 .
\end{gathered}
$$

Основным результатом данной работы является доказательство, что на самом деле $\alpha=2$, и если $\mathbf{W}$ многообразие, определенное тождествами (3), (4) и (5), то $\mathbf{W}=\operatorname{var} A$. Сформулируем соответствующее утверждение. 
Теорема 1. Многообразие $\mathbf{W}$ порождается алгеброй $A$, то есть $\mathbf{W}=\operatorname{var} A$. Характер $\chi_{n}^{Q}(\mathbf{W})$ имеет следующее строение:

$$
\chi_{2 k+1}^{Q}(\mathbf{W})=2 \chi_{(k+1, k)}, \quad \chi_{2 k}^{Q}(\mathbf{W})=2 \chi_{(k, k)}+\chi_{(k+1, k-1)}, \quad k \geq 1 .
$$

Доказательство. Как отмечалось выше, согласно результатам работы [1] в сумме (2) для многообразия $\operatorname{var} A$ ненулевые кратности задаются следующими числами: $m_{(k+1, k-1)}^{Q}(\operatorname{var} A)=1$, $m_{(k+1, k)}^{Q}(\operatorname{var} A)=2,1 \leq m_{(k, k)}^{Q}(\operatorname{var} A) \leq 2$. Так как при доказательстве этого факта строение алгебры $A$ не использовалось, а использовались только тождества (3)-(5), то аналогичное утверждение верно и для многообразия $\mathbf{W}$.

Для $n=2 k$ определим следующий элемент: $g=x_{0} \bar{x}_{1} \bar{x}_{2} \ldots \widetilde{x_{1} \widetilde{x_{2}}}=x_{0} g^{\prime}$, в котором содержится $k$ одинаковых пар $\left\{x_{1}, x_{2}\right\}$ альтернированных образующих. Заметим, что элемент $g^{\prime}$ получен из идемпотента, соответствующего следующей таблице Юнга:

$$
T_{\lambda}=\begin{array}{|c|c|c|c|}
\hline 1 & 3 & \cdots & n-1 \\
\hline 2 & 4 & \cdots & n \\
\hline
\end{array}
$$

путем отождествления образующих по каждой строке.

Если $k=2 m$, то, как показано в работе [1], элемент $g$ по модулю тождеств (3)-5 можно представить в виде линейной комбинации следующих двух элементов:

$$
\begin{aligned}
& g_{1}=x_{0} x_{1} x_{1} x_{2} x_{2} \ldots x_{1} x_{1} x_{2} x_{2}+x_{0} x_{2} x_{2} x_{1} x_{1} \ldots x_{2} x_{2} x_{1} x_{1}, \\
& g_{2}=x_{0} x_{1} x_{2} x_{2} x_{1} \ldots x_{1} x_{2} x_{2} x_{1}+x_{0} x_{2} x_{1} x_{2} x_{1} \ldots x_{2} x_{1} x_{1} x_{2} .
\end{aligned}
$$

Докажем, что элементы $g_{1}$ и $g_{2}$ являются линейно независимыми. Предположим, что $g_{1}$ и $g_{2}$ линейно зависимые. Тогда запишем их линейную комбинацию в виде $\alpha_{1} g_{1}+\alpha_{2} g_{2}=0$, где $\alpha_{1}^{2}+\alpha_{2}^{2} \neq 0$. Так как любое соотношение на свободных образующих является тождеством, то проанализируем следствия из тождества

$$
\alpha_{1} g_{1}+\alpha_{2} g_{2} \equiv 0
$$

Умножим тождество (6) дважды на $x_{1}$ справа, получим:

$$
\begin{gathered}
\alpha_{1} x_{0} x_{1} x_{1} x_{2} x_{2} \ldots x_{1} x_{1} x_{2} x_{2} x_{1} x_{1}+\alpha_{1} x_{0} x_{2} x_{2} x_{1} x_{1} \ldots x_{2} x_{2} x_{1} x_{1} x_{1} x_{1}+ \\
+\alpha_{2} x_{0} x_{1} x_{2} x_{2} x_{1} \ldots x_{1} x_{2} x_{2} x_{1} x_{1} x_{1}+\alpha_{2} x_{0} x_{2} x_{1} x_{2} x_{1} x_{2} \ldots x_{2} x_{1} x_{1} x_{2} x_{1} x_{1} \equiv 0 .
\end{gathered}
$$

Второе и третье слагаемые тождественно равны нулю как следствия тождества (4), а по тождеству (5) четвертое слагаемое также тождественно равно нулю. Поэтому если $\alpha_{1} \neq 0$, то в многообразии $\mathbf{W}$ выполнено следующее тождество:

$$
x_{0} x_{1} x_{1} x_{2} x_{2} \ldots x_{1} x_{1} x_{2} x_{2} x_{1} x_{1} \equiv 0 .
$$

Получили противоречие, так как это тождество не выполняется в алгебре $A$, которая принадлежит многообразию $\mathbf{W}$. Действительно, если подставить $x_{0}=z a, x_{1}=b, x_{2}=a$, то получим ненулевой результат, равный $(-1)^{m} z\left(R_{a} R_{b}\right)^{k} a$. Если же $\alpha_{1}=0$, то в этом случае $\alpha_{2} \neq 0$. Тождество (6) примет вид $g_{2} \equiv 0$, что также приводит к противоречию, поскольку это тождество не выполнено в алгебре $A$. Если подставить $x_{0}=z, x_{1}=a, x_{2}=b$, то получим ненулевой результат, равный $2(-1)^{m} z\left(R_{a} R_{b}\right)^{k}$.

Таким образом, элементы $g_{1}$ и $g_{2}$ являются линейно независимы. Пусть теперь $f_{1}=\operatorname{lin}\left(g_{1}\right)$, $f_{2}=\operatorname{lin}\left(g_{2}\right)$ - результаты полных линеаризаций рассматриваемых элементов. Так как характеристика поля равна нулю, то хорошо известно, что тождество (6) эквивалентно тождеству $\alpha_{1} f_{1}+\alpha_{2} f_{2} \equiv 0$. Элементы $f_{1}$ и $f_{2}$ также являются линейно независимыми. Но в этом случае из леммы 2 статьи [3] будет следовать, что $m_{(k, k)}^{Q}(\operatorname{var} A) \geq 2$.

Если $k=2 m+1$, то $g$ можно записать как линейную комбинацию следующих элементов:

$$
\begin{aligned}
& h_{1}=x_{0} x_{1} x_{1} x_{2} x_{2} \ldots x_{1} x_{1} x_{2} x_{2} x_{1} x_{2}-x_{0} x_{2} x_{2} x_{1} x_{1} \ldots x_{2} x_{2} x_{1} x_{1} x_{2} x_{1}, \\
& h_{2}=x_{0} x_{1} x_{2} x_{2} x_{1} \ldots x_{1} x_{2} x_{2} x_{1} x_{1} x_{2}-x_{0} x_{2} x_{1} x_{1} x_{2} \ldots x_{2} x_{1} x_{1} x_{2} x_{2} x_{1} .
\end{aligned}
$$

Аналогично предыдущему случаю рассматриваем тождество

$$
\alpha_{1} h_{1}+\alpha_{2} h_{2} \equiv 0 .
$$


Только в этот раз при получении следствия домножим тождество (7) на $x_{2}$ справа, а затем и на $x_{1}$ справа. Выпишем полученное тождественное соотношение:

$$
\begin{gathered}
\alpha_{1} x_{0} x_{1} x_{1} x_{2} x_{2} \ldots x_{1} x_{1} x_{2} x_{2} x_{1} x_{2} x_{2} x_{1}-\alpha_{1} x_{0} x_{2} x_{2} x_{1} x_{1} \ldots x_{2} x_{2} x_{1} x_{1} x_{2} x_{1} x_{2} x_{1}+ \\
+\alpha_{2} x_{0} x_{1} x_{2} x_{2} x_{1} \ldots x_{1} x_{2} x_{2} x_{1} x_{1} x_{2} x_{2} x_{1}-\alpha_{2} x_{0} x_{2} x_{1} x_{1} x_{2} \ldots x_{2} x_{1} x_{1} x_{2} x_{2} x_{1} x_{2} x_{1} \equiv 0 .
\end{gathered}
$$

С помощью тождества (3) представим второе слагаемое как сумму двух слагаемых

$$
\begin{gathered}
\alpha_{1} x_{0} x_{1} x_{1} x_{2} x_{2} \ldots x_{1} x_{1} x_{2} x_{2} x_{1} x_{2} x_{2} x_{1}- \\
-\alpha_{1} x_{0} x_{2} x_{2} x_{1} x_{1} \ldots x_{2} x_{2} x_{1} x_{1} x_{2} x_{1} x_{1} x_{2}+\alpha_{1} x_{0} x_{2} x_{2} x_{1} x_{1} \ldots x_{2} x_{2} x_{1} x_{1} x_{2} x_{2} x_{1} x_{1}+ \\
+\alpha_{2} x_{0} x_{1} x_{2} x_{2} x_{1} \ldots x_{1} x_{2} x_{2} x_{1} x_{1} x_{2} x_{2} x_{1}+\alpha_{2} x_{0} x_{2} x_{1} x_{1} x_{2} \ldots x_{2} x_{1} x_{1} x_{2} x_{2} x_{1} x_{1} x_{2} \equiv 0
\end{gathered}
$$

Первое и второе слагаемые тождественно равны нулю как следствия тождества (5), а третье и четвертое слагаемые при замене $x_{1}$ на $x_{2}$ приводят к тождеству

$$
\alpha_{1} g_{1}+2 \alpha_{2} g_{2} \equiv 0
$$

По ранее доказанному элементы $g_{1}$ и $g_{2}$ линейно независимы, поэтому $\alpha_{1}=0$ и $\alpha_{2}=0$. Следовательно, элементы $h_{1}$ и $h_{2}$ линейно независимы. В результате получили, что $m_{(k, k)}^{Q}(\operatorname{var} A) \geq 2$.

Осталось заметить, что $A \in \mathbf{W}$, поэтому $m_{(k, k)}^{Q}(\operatorname{var} A) \leq m_{(k, k)}^{Q}(\mathbf{W})$ и мы получаем цепочку неравенств $2 \leq m_{(k, k)}^{Q}(\operatorname{var} A) \leq m_{(k, k)}^{Q}(\mathbf{W}) \leq 2$. Окончательно получаем требуемое равенство $m_{(k, k)}^{Q}(\operatorname{var} A)=m_{(k, k)}^{Q}(\mathbf{W})=2$, а также совпадение многообразий $\mathbf{W}=\operatorname{var} A$.

Теорема 1 полностью доказана.

Перейдем к изложению полученных результатов о числовых характеристиках многообразия $\mathbf{W}$.

Теорема 2. Разложение характера $\chi_{n}(\mathbf{W})$ в сумму неприводимьх имеет вид

$$
\begin{gathered}
\chi_{2 k}(\mathbf{W})=2 \chi_{(k, k)}+2 \chi_{(k+1, k-1)}+2 \chi_{(k, k-1,1)}, \\
\chi_{2 k+1}(\mathbf{W})=2 \chi_{(k+2, k-1)}+3 \chi_{(k+1, k)}+2 \chi_{(k+1, k-1,1)} .
\end{gathered}
$$

Для кодлины многообразия $\mathbf{W}$ верны формуль $l_{2 k}(\mathbf{W})=6, l_{2 k+1}(\mathbf{W})=7$, а экспонента многообразия равна двум, $\exp \mathbf{W}=2$.

Доказательство. Доказательство того факта, что в разложении характера $\chi_{n}(\mathbf{W})$ присутствуют только такие неприводимые характеры, именно с такими кратностями следует из теории представления симметрических групп [4]. Модуль $P_{n+1}(\mathbf{W})$ индуцирован из $\Phi S_{n}$-модуля $Q_{n}(\mathbf{W})$. Тогда по теореме 1 и правилу Литтлвуда - Ричардсона получаем, что ненулевые кратности в разложении характера $\chi_{n}(\mathbf{W})$ будут только те, которые представлены в формулировке теоремы, причем со следующими ограничениями на кратности:

$$
\begin{aligned}
m_{(k, k)}(\mathbf{W}) \leq 2, & m_{(k+1, k-1)}(\mathbf{W}) \leq 2, & & m_{(k, k-1,1)}(\mathbf{W}) \leq 2 \\
m_{(k+2, k-1)}(\mathbf{W}) \leq 2, & m_{(k+1, k)}(\mathbf{W}) \leq 3, & & m_{(k+1, k-1,1)}(\mathbf{W}) \leq 2 .
\end{aligned}
$$

Кроме того, в работе [1] доказано, что $\operatorname{dim} P_{n+1}(\mathbf{W})=(n+1) \operatorname{dim} P_{n}^{Q}(\mathbf{W})$. В итоге приходим к равенствам

$$
\begin{aligned}
m_{(k, k)}(\mathbf{W})=2, & m_{(k+1, k-1)}(\mathbf{W})=2, & & m_{(k, k-1,1)}(\mathbf{W})=2 ; \\
m_{(k+2, k-1)}(\mathbf{W})=2, & m_{(k+1, k)}(\mathbf{W})=3, & & m_{(k+1, k-1,1)}(\mathbf{W})=2 .
\end{aligned}
$$

Формулы для кодлины многообразия получаются непосредственным суммированием найденных кратностей.

Используя формулу крюков (см., например, [2, с. 48]), непосредственными вычислениями находим формулы для коразмерностей:

$$
\begin{gathered}
c_{n}(\mathbf{W})=\frac{2(5 n+8)}{(n+2)(n+4)}\left(\begin{array}{c}
n \\
\frac{n}{2}
\end{array}\right) ; \\
c_{n}(\mathbf{W})=\frac{4}{(n+3)}\left(\begin{array}{c}
n \\
\frac{n-1}{2}
\end{array}\right) .
\end{gathered}
$$


Перейдем к доказательству, что экспонента многообразия $\mathbf{W}$ равна двум. Хорошо известно, что сумма биномиальных коэффициентов равна $\sum_{s=0}^{n}\left(\begin{array}{l}n \\ s\end{array}\right)=2^{n}$. Так как $n=2 k$ или $n=2 k+1$, то $\left(\begin{array}{l}n \\ k\end{array}\right)>\left(\begin{array}{l}n \\ s\end{array}\right)$ для всех других $s$, отличных от $k$, поэтому выполняются неравенства:

$$
\frac{1}{(n+1)} 2^{n} \leq\left(\begin{array}{l}
n \\
k
\end{array}\right) \leq 2^{n}
$$

Отсюда для коразмерности выполняются, например, такие неравенства:

$$
\frac{1}{(n+4)^{3}} 2^{n} \leq c_{n}(\mathbf{W}) \leq 2^{n} .
$$

$\mathrm{C}$ помощью сведений из математического анализа получаем, что $\exp \mathbf{W}=2$. Теорема 2 доказана.

Выражаю благодарность моему научному руководителю, доктору физико-математических наук, профессору Сергею Петровичу Мищенко за постановку задачи, полезные советы, постоянное внимание к работе и всестороннюю поддержку.

\section{Библиографический список}

1. Mishchenko S., Valenti $A$. An almost nilpotent variety of exponent 2 // Israel J. of Math. 2014. Vol. 199, iss. 1. P. 241-257.

2. Giambruno A., Zaicev M. Polynomial Identities and Asymptotic Methods. Math. Surv. and Monographs. Vol. 122. Providence, RI : Amer. Math. Soc., 2005. 352 p.

3. Зайцев М. В., Мищенко С. П. О кодлине многообразий линейных алгебр // Мат. заметки. 2006. Т. 79, вып. 4. С. 553-559. DOI: 10.4213/mzm2724.

4. Джеймс Г. Теория представлений симметрических групп. М. : Мир, 1982. 214 с.

\section{New Properties of Almost Nilpotent Variety of Exponent 2}

\section{O. V. Shulezhko}

Ulyanovsk State University, 42, Leo Tolstoy str., Ulyanovsk, 432970, Russia, ol.shulezhko@gmail.com

In the presented work we consider numerical characteristics of almost nilpotent variety of exponent 2, which was first constructing in article [1]. The main result of this paper is introduce the exact values of the multiplicities of the irreducible modules appearing in the expansion of the multilinear part of the variety. Meanwhile, we obtain as a consequence the formulas of codimension and colength of the variety of exponent 2 .

Key words: variety, exponent of variety, codimension, colength.

\section{References}

1. Mishchenko S., Valenti A. An almost nilpotent variety of exponent 2. Israel J. of Math., 2014, vol. 199, iss. 1, pp. 241-257.

3. Zaitsev M. V., Mishchenko S. P. Colength of varieties of linear algebras. Math. Notes, 2006, vol. 79, no. 4, pp. 511-517. DOI: 10.1007/s11006-006-0056-0.

2. Giambruno A., Zaicev M. Polynomial Identities and 4. James G. D. The representation theory of the symmetAsymptotic Methods. Math. Surv. and Monographs, ric groups. Lecture Notes in Math., vol. 682, Berlin, New vol. 122, Providence, RI, Amer. Math. Soc., 2005, 352 p. York, Springer-Verlag, 1978. 